Research Article

\title{
Wavelet Bilateral Filter Algorithm-Based High-Frequency Ultrasound Image Analysis on Effects of Skin Scar Repair
}

\author{
Zhidong Xuan (D), Haixia Liu $(\mathbb{D}$, Chao Li $(\mathbb{D}$, and Yongrong Liu $(\mathbb{D}$ \\ Department Three of Ultrasound, Cangzhou Central Hospital, No. 16 Xinhua Road, Cangzhou 061001, Hebei, China \\ Correspondence should be addressed to Zhidong Xuan; 13261102@bjtu.edu.cn
}

Received 17 June 2021; Accepted 22 July 2021; Published 3 August 2021

Academic Editor: Gustavo Ramirez

Copyright (c) 2021 Zhidong Xuan et al. This is an open access article distributed under the Creative Commons Attribution License, which permits unrestricted use, distribution, and reproduction in any medium, provided the original work is properly cited.

\begin{abstract}
It aimed to explore the application value of high-frequency ultrasound image based on the wavelet bilateral filtering algorithm in the repair evaluation of hypertrophic scar and keloid, as well as the expression change of nerve growth factor (NGF). 72 patients with scars admitted to hospital from October 2018 to October 2019 were chosen and classified as hypertrophic scar patients (group A) and keloid patients (group B), with 36 cases in each group. All patients received scar repair treatment, and another 30 normal skin volunteers (group C) were selected. High-frequency ultrasound images based on the wavelet bilateral filter algorithm were utilized for skin examination. The expression differences of NGF and pro-NGF in each group were measured by immunohistochemical staining and Western blot. The results showed that the denoising effect (signal-to-noise ratio $($ PSNR) $=33.2762$ ), structural similarity $($ SSIM $=0.8963)$, and edge similarity $(\mathrm{FoM}=0.2975)$ of the proposed algorithm were better than those of bilateral filtering and wavelet soft-threshold algorithms. The skin thickness of groups A and B was considerably higher relative to that of group $\mathrm{C}(P<0.05)$, but the echo intensity of the skin dermis of groups A and B was evidently inferior to that of group $\mathrm{C}$ $(P<0.05)$. The results of immunohistochemistry showed that in contrast to group $\mathrm{C}$, the average optical density of NGF in groups $\mathrm{A}$ and $\mathrm{B}$ increased remarkably $(P<0.01)$. In contrast to group $\mathrm{C}$, the positive expression of pro-NGF in groups $\mathrm{A}$ and $\mathrm{B}$ was notably reduced $(P<0.05)$. Western blot results showed that relative to group $\mathrm{C}$, the expression of NGF protein in groups A and $\mathrm{B}$ was increased greatly $(P<0.01)$, but the expression of pro-NGF protein in groups A and B was reduced $(P<0.01)$. The results suggested that the wavelet bilateral filter algorithm can be adopted to evaluate pathological skin scars, and pathological skin scar formation was closely related to the expression levels of NGF and pro-NGF.
\end{abstract}

\section{Introduction}

The result of excessive tissue fibrosis after skin trauma is skin scar [1]. Skin scars are usually accompanied by symptoms of itching or pain and can also affect the appearance and greatly affect the quality of life of patients. Pathological skin scars mainly include hypertrophic scars and keloids [2,3]. Scar hyperplasia and contracture will also greatly reduce the patient's joint mobility. In severe cases, it can lead to joint deformities, which can lead to limb dysfunction. Pathological scar formation is related to many factors. The research on neuropeptides and scar hyperplasia is a hot spot in the field of pathological scars. Studies have found that NGF plays an important role in wound repair [4]. NGF can effectively promote the gradual migration of vascular endothelial cells and immune cells to the wounded part. Then, it can promote inflammatory cell infiltration and finally play a positive role in promoting the proliferation of new epithelial cells and blood vessels. Massive studies have shown that NGF plays a biological role in the production of pathological scars [5], but further research and verification are needed.

At present, the Vancouver Scar Assessment Scale [6] is widely adopted to assess scars clinically. Most scales that are widely adopted to assess scars are subjectively based on clinical observations, and it is difficult to objectively and accurately evaluate the scars, so there is an increasing need to find a noninvasive objective tool for accurate diagnosis and evaluation of scars. With the rapid progression of medical imaging diagnostic technology, high-frequency ultrasound has become the main diagnosis tool due to its higher resolution and penetration rate, which is able to diagnose and distinguish the fine structure of the skin $[7,8]$. High- 
frequency ultrasound refers to the frequency of the ultrasound probe exceeding $10 \mathrm{MHz}$, which can not only perform real-time imaging of tissues in vivo but also detect skin morphology and physiological structure, with high safety and is noninvasive. Especially, $20 \mathrm{MHz}$ high-frequency ultrasound is widely adopted in the diagnosis of skin lesions. The skin surface, dermis, and subcutaneous tissues can be clearly distinguished. However, there are few reports about pathological scars. In medical ultrasound images, speckle noise has a greater impact on image quality. The wavelet transform denoising algorithm is a commonly used ultrasound image denoising algorithm [9], but it is very susceptible to changes in the noise frequency distribution, and speckle noise is still present in the low-frequency part. The bilateral filter can suppress the low-frequency domain noise, while also effectively retaining the edge information of the ultrasound image [10].

Therefore, the two methods were combined, and a medical ultrasound denoising algorithm based on wavelet bilateral filter was proposed. It was applied to high-frequency ultrasound image processing to obtain high-quality images, to evaluate pathological skin scars, and to measure the expression changes of NGF and its pro-NGF, hoping to provide a theoretical basis for the diagnosis and prevention of pathological scars.

\section{Materials and Methods}

2.1. Research Objects and Grouping. 72 patients with scars admitted to hospital from October 2018 to October 2019 were selected as the research objects. According to the different pathological types of scars, they were divided into hypertrophic scar patients (group A) and keloid patients (group B), with 36 cases in each group. Then, 30 normal skin volunteers (group C) were selected. All subjects had no history of chronic skin diseases and had been treated with scar repair. Moreover, patients who were allergic to ultrasound gel were excluded. This experiment had been approved by the ethics committee of the hospital, and the patients included in the study had known the whole process and agreed with it.

2.2. High-Frequency Ultrasonic Detection. Before the examination, the skin of the subject's scar was cleaned, and then high-frequency ultrasound examination was started. First, a professional doctor applied a special ultrasound gel to the center of the scar. After which, the skin ultrasound probe would be operated. The probe frequency was $20 \mathrm{MHz}$, perpendicular to the skin surface of the subject. It gently touched the subject's skin, and the probe should be controlled to avoid pressure on it. Three pictures for each part were collected, the thickness of the three scars in each picture was measured, and the average of the results was taken. Then, the ultrasound images of normal skin and scar skin were input into the DFY-1 ultrasound image diagnosis and analysis software, to analyze the ultrasound images of the scar. Three areas for each picture were selected to measure the echo intensity, and the average of the results was taken.

2.3. Ultrasound Image Algorithm Based on Wavelet Bilateral Filtering. The traditional wavelet denoising algorithm performs wavelet coefficient filtering processing on the highfrequency domains of LH, HL, and $\mathrm{HH}$ but does not process the low-frequency part of LL. However, it was found that there was still speckle noise in the low-frequency part of the wavelet domain, and speckle noise mainly presented large particles in medical ultrasound images. Therefore, this work combined a bilateral filter to suppress low-frequency noise, while also effectively retaining the edge information of ultrasound images.

The threshold processing and shrinking algorithm for wavelet decomposition of high-frequency sub-bands are equations (1) and (2):

$$
F=\frac{1}{2^{j-1}}\left(k_{1} \sigma_{\mathrm{n}} \sqrt{2 \log M}+k_{2} \frac{\sigma_{n}^{2}}{\sigma_{g \cdot j}}\right) .
$$

In equation (1), $\sigma_{n}$ is the noise standard deviation; $\sigma_{g \cdot j}$ is the noise-free image standard deviation; $M$ is the total number; $F$ is the threshold function; $k_{1}$ and $k_{2}$ are parameters; $j$ is the number of layers; and $g$ is the noise-free signal.

$$
\widehat{g}= \begin{cases}0, & f \leq F_{j}, \\ \operatorname{sign}(f) \cdot \max \left(|f|-\frac{\sigma_{n}^{2}}{s}, 0\right), & f>F_{j} .\end{cases}
$$

In equation (2), $s$ is the scale parameter; $\hat{g}$ is the estimate of $g$; and $f$ is the input image.

Then, the lowest frequency sub-band is subjected to bilateral filtering, and the parameters of the bilateral filter are set as follows. Gaussian kernel is selected as the regional filter kernel (equation (3), and Gaussian kernel is selected as the kernel of the range filter (equation (4)).

$$
\begin{gathered}
c(\xi, x)=\exp \left(-\frac{(\|\xi-x\|)^{2}}{2 \sigma_{c}^{2}}\right), \\
s(f(\xi), f(x))=\exp \left(-\frac{(\|\| \xi-x|| \|)^{2}}{2 \sigma_{s}^{2}}\right) .
\end{gathered}
$$

In the above equation, $x$ is the central pixel; $c(\xi, x)$ is the closeness function; $s(f(\xi), f(x))$ is the similarity function; $\|\cdot\|$ is the Euclidean distance; $\sigma_{c}$ is the standard deviation of the Gaussian kernel of the closeness function; and $\sigma_{s}$ is the standard deviation of the Gaussian kernel of the similarity function.

In this article, $\sigma_{c}$ is 2.35 and $\sigma_{s}$ is 0.1 . The window size is $10 \times 10$ pixels, the maximum number of decomposition layers is set to 4 , and the "coifl" wavelet base is taken as the two-dimensional discrete wavelet base. 
The peak signal-to-noise ratio (PSNR), structural similarity (SSIM), FoM, and time indicators were adopted to evaluate the quality of ultrasound images.

$$
\begin{aligned}
\operatorname{PSNR}(X, \hat{X}) & =10 \log _{10}\left(\frac{255^{2}}{\operatorname{MSE}}\right), \\
\mathrm{MSE} & =\frac{1}{M N} \sum_{i=1}^{M} \sum_{j=1}^{N}\left(X_{i, j}-\widehat{X}_{i, j}\right)^{2} .
\end{aligned}
$$

In equation (5), $X$ is the two-dimensional signal; $M$ is the length of the two-dimensional signal; $N$ is the width of the two-dimensional signal; and $\widehat{X}$ is the estimated value of $X$.

$$
\operatorname{SSIM}(X, \widehat{X})=\frac{\left(2 \mu_{x} \mu_{\hat{x}}+c_{1}\right)\left(2 \sigma_{x \hat{x}}+c_{2}\right)}{\left(\mu_{x}^{2}+\mu_{\hat{x}}+c_{1}\right)\left(\sigma_{x}^{2}+\sigma_{\hat{x}}^{2}+c_{2}\right)}
$$

In equation (6), $c_{1}$ and $c_{2}$ are constants; $\sigma_{x}^{2}$ is the reference image variance; $\sigma_{\widehat{x}}^{2}$ is the estimated image variance; and $\sigma_{x \hat{x}}$ is the covariance. SSIM has a value range of $0-1$. The closer the result is to 1 , the more identical the structure of the two images.

$$
\operatorname{FoM}(X, \hat{X})=\frac{1}{\max \left(N_{X}, N_{\widehat{X}}\right)} \sum_{i=1}^{N_{\widehat{X}}} \frac{1}{1+\alpha d_{i}^{2}} .
$$

In equation (7), $\alpha$ is a constant and $N_{X}$ and $N_{\widehat{X}}$ are the pixel numbers of the ideal edge and the actual edge, respectively. The value of FoM is in the range of $0-1$. The closer the result is to 1 , the closer the edge of the detected image to the ideal edge.

\subsection{Immunohistochemical Staining (Strept Avidin-Biotin} Complex (SABC) Method). Firstly, tissue sections were prepared and treated with antigen retrieval and then soaked in PBS buffer solution for three times, $5 \mathrm{~min}$ each. After which, $3 \% \mathrm{H}_{2} \mathrm{O}_{2}$ deionized water was added, and sections were placed in a wet box at $37^{\circ} \mathrm{C}$ for $30 \mathrm{~min}$ for incubation. Then, PBS buffer solution was adopted to soak sections for three times, $5 \mathrm{~min}$ each. $10 \%$ goat seal serum and primary and secondary antibody diluent were added. DAB solution was adopted for color development, and sections were observed under a microscope, and tap water was adopted for termination of staining. After added with hematoxylin, sections were placed for $5 \mathrm{~min}$, rinsed with running water, divided $2 \mathrm{~s}$ with $1 \%$ hydrochloric acid alcohol, which was repeated for 3-5 times, and then sections were rinsed with running water for $5 \mathrm{~min}$. $75 \%, 85 \%$, and $95 \%$ ethanol were utilized for dehydration for $60 \mathrm{~s}$, respectively, and then sections were treated with anhydrous ethanol for $4 \mathrm{~min}$. The final staining results were observed under the microscope after neutral gum was added to the slide. If the cytoplasm or membrane was brownish yellow, the expression was positive. Image-pro Plus6.0 software was adopted to detect NGF expression, and the optical density value was recorded. ProNGF expression was analyzed by calculating the proportion of positive cells in the total number of cells, which was classified as $>50 \%, 25 \%-50 \%, 10 \%-25 \%$, and $<10 \%$, which was corresponding to the four grades ",,,++++++- ," respectively, where ++ and +++ were high expression while - and + were low expression.

2.5. Western Blot. Western blot was adopted to detect the protein expression levels of NGF and pro-NGF. $150 \mathrm{mg}$ of tissue was taken, cleaned, placed in EP tube, cut into small pieces, and treated with homogenizer until the tissue block disappeared. About $100 \mu \mathrm{L}$ of RIPA lysis buffer was added and placed on ice for $30 \mathrm{~min}$ for cracking. Then, it was removed and placed in an environment of $4^{\circ} \mathrm{C}$ and centrifuged for $30 \mathrm{~min}, 20000 \mathrm{r} / \mathrm{min}$. The supernatant was absorbed and transferred to a new EP tube. The BCA method was adopted to detect the protein concentration. 10\% SDS polyacrylamide gel was prepared, and an appropriate amount of SDS-PAGE loading buffer was added to the $60 \mu \mathrm{L}$ sample to be tested. The protein was fully denatured in boiling water for $10 \mathrm{~min}$. After cooling, the sample to be tested and protein marker were added into the sampling hole for electrophoresis. First, $30 \mathrm{~V}$ electrophoresis was conducted for $60 \mathrm{~min}$ and then $100 \mathrm{~V}$ was conducted. After electrophoresis, PVDF membrane was utilized for protein transfer. After the membrane was transferred, 5\% milk powder blocking solution was added for $120 \mathrm{~min}$ at room temperature. The sample was placed in the primary antibody and shaken overnight at $4^{\circ} \mathrm{C}$. After the primary antibody was recovered the next day, the membrane was placed in TBST solution and shaken for $10 \mathrm{~min}$ at room temperature. The membrane was washed for 3 times. The secondary antibody was added and combined on the shaker for 1 hour. Finally, the membrane was placed in TBST solution and shaken for $10 \mathrm{~min}$ at room temperature. The membrane was washed for 3 times. Development analysis was performed using LAS4000 gel imager, and Multi Gauge V3.2 software was adopted for stripe gray analysis to obtain the relative expression of NGF and pro-NGF proteins.

2.6. Statistical Analysis. SPSS20.0 statistical software was utilized for analysis, and measurement data were expressed as mean \pm standard deviation $(\bar{x} \pm s)$, tested by the $t$-test or $\chi^{2}$ test. One-way analysis of variance was adopted to compare scar thickness and echo intensity. If $P<0.05$, the difference was statistically significant.

\section{Results}

3.1. Ultrasound Image Quality Evaluation Based on Wavelet Bilateral Filter Algorithm. PSNR, SSIM, FoM, and time index were adopted to evaluate the ultrasound image quality, as shown in Table 1. Compared with bilateral filtering and wavelet soft-threshold, the PSNR, SSIM, and FoM values of the proposed algorithm were all the largest, suggesting that the denoising effect, structural similarity, and edge similarity of the algorithm proposed in this work were favorable. However, this algorithm had no advantage in running time, and reducing complexity was where the work needed to be improved in the future. 
TABle 1: Performance comparison of different denoising algorithms.

\begin{tabular}{lcccc}
\hline Algorithm & FoM & SSIM & PSNR & $\begin{array}{c}\text { Running } \\
\text { time }(\mathrm{s})\end{array}$ \\
\hline $\begin{array}{l}\text { Bilateral filtering } \\
\text { Wavelet soft- }\end{array}$ & 0.2712 & 0.6895 & 27.9872 & 7.7920 \\
threshold & 0.1902 & 0.7354 & 25.3543 & 1.7234 \\
Proposed algorithm & 0.2975 & 0.8963 & 33.2762 & 9.5436 \\
\hline
\end{tabular}

3.2. Ultrasound Image Characteristics of Each Group. The ultrasound images based on the wavelet bilateral filter algorithm were adopted to examine the skin of each group of subjects, and the results are shown in Figure 1. The thickness of the skin in group $\mathrm{C}$ was relatively thin, and the echo intensity of the skin dermis was uniform. The skin of group A and group B was thicker, and the echo intensity of the dermis layer was low echo. Among them, the hypoechoic zone of group A was a hypoechoic zone in the upper layer of the dermis, and the hypoechoic zone of group B was an oval hypoechoic area under the epidermis. From the perspective of organizational arrangement, the organizational structure of each level of group C was very complete and the boundaries are relatively clear. The boundaries between the dermis and subcutaneous tissues of group A and group B were clear, and the epidermis was uneven, showing an upward bulge.

3.3. Comparison of Scar Skin Thickness and Echo Intensity. Table 2 shows the comparison results of skin thickness and dermal echo intensity of each group. The skin thickness of group A was $4.48 \pm 0.45(\mathrm{~mm})$, group B was $4.23 \pm 0.23$ $(\mathrm{mm})$, and group $C$ was $1.92 \pm 0.11(\mathrm{~mm})$. The skin thickness of groups $\mathrm{A}$ and $\mathrm{B}$ was remarkably higher relative to group $\mathrm{C}$ $(P<0.05)$. There was no notable difference in skin thickness between groups $\mathrm{A}$ and $\mathrm{B}(P>0.05)$. The echo intensity of the dermis of group A was $40.36 \pm 10.23$, group B was $17.23 \pm 7.25$, and group $C$ was $95.27 \pm 20.12$. The echo intensity of the skin dermis of groups B and A was evidently inferior to that of group $\mathrm{C}(P<0.05)$. The echo intensity of the skin dermis of group $\mathrm{B}$ was obviously lower in contrast to that of group A $(P>0.05)$.

3.4. Immunohistochemical Staining Results. The immunohistochemical results of NGF and pro-NGF expression in each group are shown in Figures 2 and 3. NGF was mainly expressed in the epidermis and dermis. A large number of positive particles could be observed in the epidermal basal layer and dermal fibroblasts in groups A and B. In group C, the positive expressions were mostly distributed in the basal layer of the epidermis and near the skin appendages. The expression of pro-NGF was positive in the three groups A, B, and $\mathrm{C}$, which was mainly expressed in the epidermis and fibroblasts of tissues. Table 3 shows the comparison of the average optical density of NGF and the proportion of proNGF positive expression in each group. In contrast to group $\mathrm{C}$, the average optical density of NGF in groups A and B increased considerably $(P<0.01)$. Compared with group $\mathrm{C}$, the positive expression of pro-NGF in groups A and B was substantially reduced $(P<0.05)$.

3.5. NGF and Pro-NGF Protein Expression. The protein expression levels of NGF and pro-NGF in each group were determined by Western blot, and the results are shown in Figure 4. The expression levels of NGF protein in groups A, $B$, and $C$ were $2.57 \pm 0.43,2.43 \pm 0.25$, and $1.30 \pm 0.09$, respectively. The pro-NGF protein expression in groups $\mathrm{A}, \mathrm{B}$, and $C$ was $1.59 \pm 0.35,1.47 \pm 0.25$, and $2.62 \pm 0.23$, respectively. Compared with group $\mathrm{C}$, the expression of NGF protein in groups A and B was increased notably $(P<0.01)$, and the expression of pro-NGF protein was decreased substantially $(P<0.01)$.

\section{Discussion}

In this research, the wavelet bilateral filter denoising algorithm was applied to ultrasonic image processing. The results showed that the denoising effect $(\mathrm{PSNR}=33.2762)$, structural similarity $($ SSIM $=0.8963)$, and edge similarity $(\mathrm{FoM}=0.2975)$ were better than bilateral filtering and wavelet soft-threshold algorithms. It was consistent with the research results of Majeeth and Babu [11], showing that the combination of the wavelet transform algorithm and bilateral filtering algorithm can improve the quality of medical ultrasound images. Ultrasound is a noninvasive imaging technique that relies on the measurement of sound waves reflected in human tissues. At lower frequencies, deeper structures in the body can be seen, such as internal organs. However, the tissue penetration depth of high-frequency ultrasound with a transducer frequency of $20 \mathrm{MHz}$ or higher is much lower, yet it will produce higher resolution images, such as tissues and structures close to the skin surface [12]. Elrefaie et al. compared the Vancouver Scar Scale (VSS) and clinical assessment scoring system, to analyze the value of high-resolution ultrasound for scar assessment. It was found that after three courses of laser treatment, the Vancouver Scar Scale score significantly decreased, ultrasound showed a notable improvement in lesion thickness and echogenicity, and there was evident difference between the percentage of improvement measured by VSS and high-resolution ultrasound $(P=0.001)[13]$. The results of this study showed that the skin thickness of groups $\mathrm{A}$ and $\mathrm{B}$ was considerably higher relative to that of group $\mathrm{C}(P<0.05)$. On the contrary, the echo intensity of skin dermis in groups $\mathrm{A}$ and $\mathrm{B}$ was remarkably inferior to that of group C $(P<0.05)$. The ultrasound imaging characteristics of hypertrophic scars and keloids were characterized by increased skin thickness, and the echo intensity of the dermis of the skin was weakened, which was consistent with the results of Elrefaie's study.

Effective healing of skin wounds is critical to ensure the skin's vital barrier function, but pathologic wound healing and scar formation are major medical problems that pose both physical and psychological challenges to patients. The healing process involves many closely coordinated regenerative reactions, including hemostasis, migration of various cell types to wounds, inflammation, angiogenesis, and 


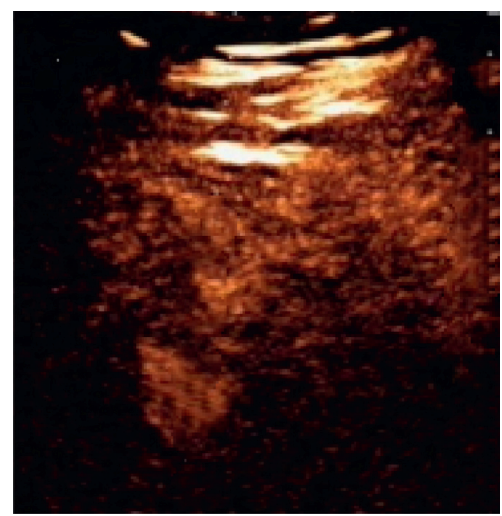

(a)

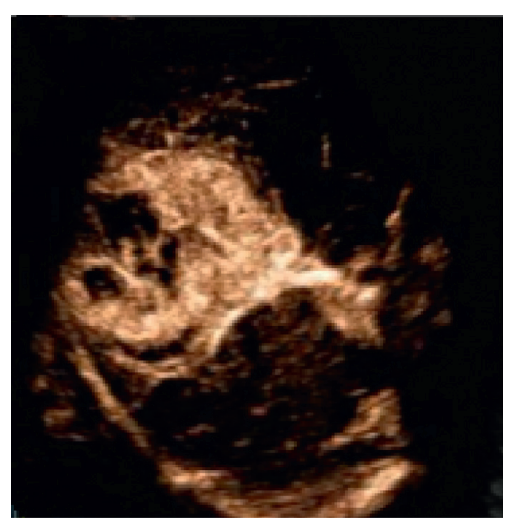

(b)

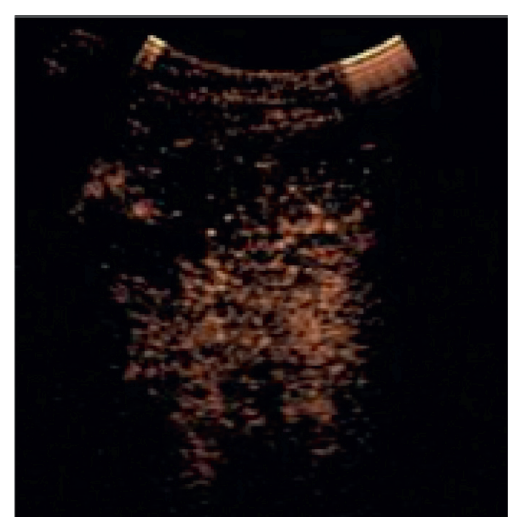

(c)

FIgURE 1: Features of ultrasound images of some patients: (a) patient in group A; (b) patient in group B; (c) patient in group C.

TABle 2: Comparison of skin thickness and dermal echo intensity in each group.

\begin{tabular}{lccc}
\hline & A & B & C \\
\hline Skin thickness $(\mathrm{mm})$ & $4.48 \pm 0.45^{*}$ & $4.23 \pm 0.23^{*}$ & $1.92 \pm 0.11$ \\
Dermal echo intensity & $40.36 \pm 10.23^{* \#}$ & $17.23 \pm 7.25^{*}$ & $95.27 \pm 20.12$ \\
\hline
\end{tabular}

Note. ${ }^{*}$ means $P<0.05$ relative to group $\mathrm{C}$; ${ }^{\#}$ means $P<0.05$ relative to group $\mathrm{B}$.

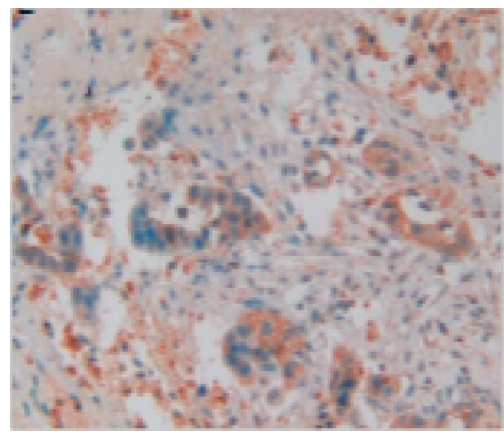

(a)

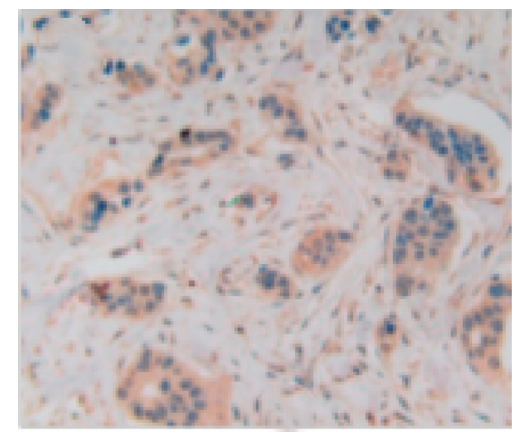

(b)

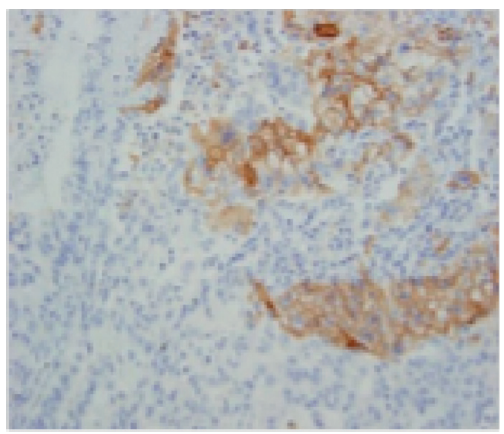

(c)

FIGURE 2: Immunohistochemical staining results of NGF expression in each group (SABC, $\times 200$ ).

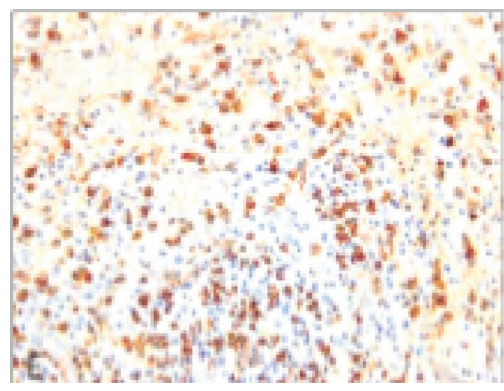

(a)

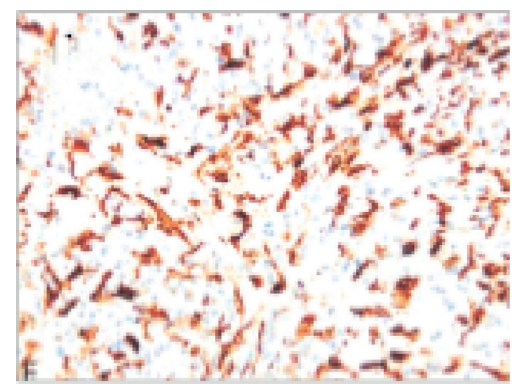

(b)

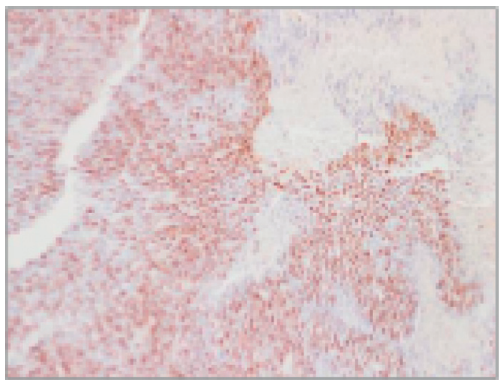

(c)

FIGURE 3: Immunohistochemical staining results of pro-NGF expression in each group (SABC, $\times 200)$.

TABLE 3: Comparison of the average optical density of NGF and the proportion of pro-NGF positive expression in each group.

\begin{tabular}{lccc}
\hline & A & B & C \\
\hline NGF average optical density & $0.1869 \pm 0.0123^{* *}$ & $0.1764 \pm 0.0119^{* *}$ & $0.0179 \pm 0.0013$ \\
Pro-NGF positive expression (\%) & $22.3^{*}$ & $19.8^{*}$ & 64.3 \\
\hline
\end{tabular}

Note. ${ }^{*}$ and ${ }^{* *}$ mean $P<0.05$ and $P<0.01$ compared with group $\mathrm{C}$, respectively. 


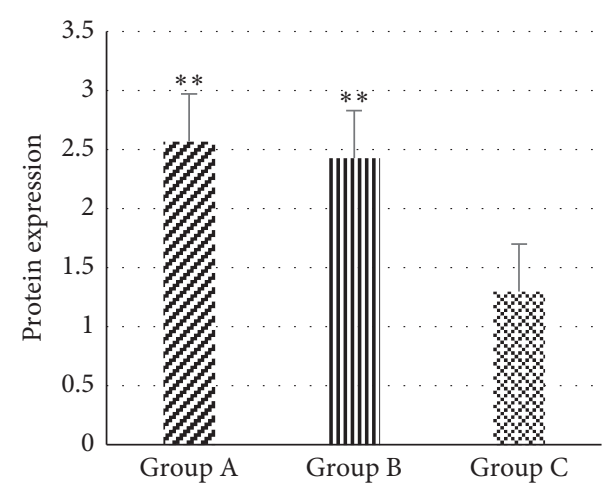

(a)

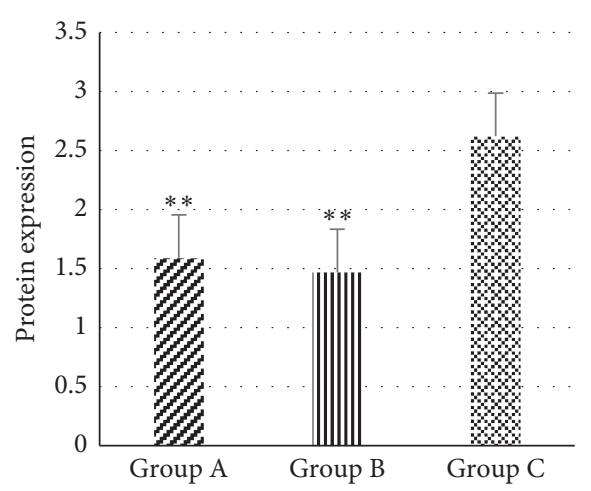

(b)

Figure 4: (a) NGF and (b) pro-NGF protein expression in each group (** means relative to group C; $P<0.01$ ).

extracellular matrix formation [14]. NGF is the first neuronutrient discovered, which is more widely distributed in nerve tissue, fibroblasts, and heart. Moreover, pro-NGF is present in many tissues [15]. The results of this study showed that NGF expression in patients with pathological scar was higher than that in normal skin. NGF is mainly expressed in the epidermis and dermis, and a large number of positive particles can be seen in the aggregation sites of epidermal basal layer and dermis fibroblasts. Studies have shown that NGF can promote migration, proliferation, and activation of fibroblasts and accelerate the secretion of fibroblasts to produce collagen [16]. This work also obtained the consistent result. It was found that pro-NGF was positively expressed in groups $\mathrm{A}, \mathrm{B}$, and $\mathrm{C}$, mainly in tissue epidermis and fibroblasts. Compared with group $\mathrm{C}$, the positive NGF expression in groups A and B was decreased $(P<0.05)$. Pro-NGF was also synthesized by epidermal and fibroblast cells. According to the difference of pro-NGF amount, it was inferred that the expression of pro-NGF decreased in pathological scar skin of groups $A$ and B. Furthermore, it may affect the normal proliferation and apoptosis of fibroblasts, which may be an important cause of the abnormal fibroblasts.

\section{Conclusion}

The wavelet bilateral filter algorithm was utilized to improve the image quality of high-frequency images and to evaluate the repair effect of pathological skin scars (hypertrophic scars and keloids). It was found that the denoising effect (PSNR $=33.2762)$, structural similarity $(\mathrm{SSIM}=0.8963)$, and edge similarity $(\mathrm{FoM}=0.2975)$ were better than bilateral filtering and wavelet soft-threshold algorithms. In contrast to normal skin, the expression of NGF protein was obviously increased in hypertrophic scars and keloids, while the expression of pro-NGF protein was decreased. The results showed that pathological skin scar formation was closely related to NGF and pro-NGF expression levels. However, the wavelet filter algorithm in this study still has some deficiencies. For example, the operation time is long and the number of samples is limited. Furthermore, the effect of pro-NGF on fibroblasts has not been discussed, and further optimization of the algorithm is needed in the future. The sample size will be expanded and further the effects of pro-NGF on fibroblasts will be discussed. In conclusion, the results of this study can provide reference for the diagnosis of pathological scar and the study of relative biological mechanism.

\section{Data Availability}

The data used to support the findings of this study are available from the corresponding author upon request.

\section{Conflicts of Interest}

The authors declare that they have no conflicts of interest.

\section{References}

[1] M. Monavarian, S. Kader, S. Moeinzadeh, and E. Jabbari, "Regenerative scar-free skin wound healing," Tissue Engineering Part B Reviews, vol. 25, no. 4, pp. 294-311, 2019.

[2] Q. Li, C. Zhang, and X. Fu, "Will stem cells bring hope to pathological skin scar treatment?" Cytotherapy, vol. 18, no. 8, pp. 943-956, 2016.

[3] X. X. Zhai, Z. M. Tang, J. C. Ding, and X. L. Lu, "Expression of TGF- $\beta 1 / \mathrm{mTOR}$ signaling pathway in pathological scar fibroblasts," Molecular Medicine Reports, vol. 15, no. 6, pp. 3467-3472, 2017.

[4] N. Gostynska, M. Pannella, M. L. Rocco, L. Giardino, L. Aloe, and L. Calzà, "The pleiotropic molecule NGF regulates the in vitro properties of fibroblasts, keratinocytes, and endothelial cells: implications for wound healing," American Journal of Physiology - Cell Physiology, vol. 318, no. 2, pp. C360-C371, 2020.

[5] H. D. Fu, S. Wang, B. Ge, L. Q. Li, H. M. Zeng, and Q. F. Shu, "Nerve growth factor and substance $\mathrm{P}$ may be involved in moist exposed burn ointment-mediated chronic refractory wound healing," Experimental and Therapeutic Medicine, vol. 16, no. 3, pp. 1987-1993, 2018.

[6] M. N. Busche, A. J. Thraen, A. Gohritz, H. O. Rennekampff, and P. M. Vogt, "Burn scar evaluation using the Cutometer ${ }^{\circledR}$ MPA 580 in comparison to patient and observer scar assessment scale and vancouver scar scale," Journal of Burn Care and Research, vol. 39, no. 4, pp. 516-526, 2018. 
[7] A. K. Bhatta, U. Keyal, and Y. Liu, "Application of high frequency ultrasound in dermatology," Discovery Medicine, vol. 26, no. 145, pp. 237-242, 2018.

[8] R. L. Bard, "High-frequency ultrasound examination in the diagnosis of skin cancer," Dermatologic Clinics, vol. 35, no. 4, pp. 505-511, 2017.

[9] D. Gradolewski, G. Magenes, S. Johansson, and W. J. Kulesza, "A wavelet transform-based neural network denoising algorithm for mobile phonocardiography," Sensors, vol. 19, no. 4, p. 957, 2019.

[10] H. H. Chang, Y. J. Lin, and A. H. Zhuang, "An automatic parameter decision system of bilateral filtering with GPUbased acceleration for brain MR images," Journal of Digital Imaging, vol. 32, no. 1, pp. 148-161, 2019.

[11] S. S. Majeeth and C. N. K. Babu, "Gaussian noise removal in an image using fast guided filter and its method noise thresholding in medical healthcare application," Journal of Medical Systems, vol. 43, no. 8, 2019.

[12] J. Dinnes, J. Bamber, and N. Chuchu, "Cochrane skin cancer diagnostic test accuracy group. High-frequency ultrasound for diagnosing skin cancer in adults," Cochrane Database of Systematic Reviews, vol. 12, no. 12, Article ID CD013188, 2018.

[13] A. M. Elrefaie, R. M. Salem, and M. H. Faheem, "Highresolution ultrasound for keloids and hypertrophic scar assessment," Lasers in Medical Science, vol. 35, no. 2, pp. 379-385, 2020.

[14] S. M. Karppinen, R. Heljasvaara, D. Gullberg, K. Tasanen, and T. Pihlajaniemi, "Toward understanding scarless skin wound healing and pathological scarring," F1000 Research, vol. 8, 2019.

[15] M. Fahnestock and A. Shekari, "ProNGF and neurodegeneration in alzheimer's disease," Frontiers in Neuroscience, vol. 13, 2019.

[16] Z. Xu, X. Zheng, and J. Zheng, "Tumor-derived exosomes educate fibroblasts to promote salivary adenoid cystic carcinoma metastasis via NGF-NTRK1 pathway," Oncology Letters, vol. 18, no. 4, pp. 4082-4091, 2019. 